\title{
Magnetic bead-based salivary peptidome profiling for periodontal-orthodontic treatment
}

\author{
Jieni Zhang ${ }^{1 \dagger}$, Shaonan Zhou ${ }^{1 \dagger}$, Ruoxuan $\mathrm{Li}^{3}$, Tian Cao ${ }^{1}$, Hui Zheng ${ }^{1}$, Xuedong Wang ${ }^{1}$, Yanheng Zhou ${ }^{1}$, Ning Du², \\ Feng Chen ${ }^{2^{*}}$ and Jiuxiang Lin ${ }^{1 *}$
}

\begin{abstract}
Background: Patients with periodontitis seek periodontal-orthodontic treatment to address certain functional and aesthetic problems. However, little is known of the effect of periodontitis on orthodontic treatment. Thus, we compared the differences in peptide mass fingerprints of orthodontic patients with and without periodontitis by MALDI-TOF MS using a magnetic bead-based peptidome analysis of saliva samples. In this way, we aimed to identify and explore a panel of differentially-expressed specific peptides.
\end{abstract}

Results: Saliva samples from 24 patients (eight orthodontic patients without periodontitis, eight with periodontitis and another eight with periodontitis but no orthodontic treatment) were analyzed, and peptide mass fingerprints were created by scanning MS signals using matrix-assisted laser desorption/ionization time-of-flight mass spectrometry (MALDI-TOF MS) combined with magnetic beads. Nine mass peaks showed significant differences. Orthodontic patients in the group without periodontal disease showed higher mass peaks for seven peptides of the nine, whereas the mass peaks for the other two peptides were higher in the periodontal-orthodontic patients. Besides, these differentially-expressed peptides were sequenced.

Conclusions: The elucidated candidate biomarkers indicated interactions between periodontal condition and orthodontic treatment and their contributions to the changes of saliva protein profiles. Our results provide novel insight into the altered salivary protein profile during periodontal-orthodontic treatment, and may lead to the development of a therapeutic monitoring strategy for periodontics and orthodontics.

Keywords: Periodontal-orthodontic treatment, Peptidome, Saliva, MALDI-TOF MS

\section{Background}

Periodontal-orthodontic treatment is usually needed to correct malocclusions, and may also improve periodontal health [1]. Patients who have chronic periodontal problems want to improve not only their malocclusions to obtain a perfect facial and tooth profile with orthodontic treatment, but also their periodontal condition. However, some doctors believe that orthodontic treatment aggravates periodontal damage [2]; the matter remains disputed. The difference in osteogenesis between periodontitis and non-periodontitis patients [3] may lead to differences in

\footnotetext{
*Correspondence: moleculecf@gmail.com; jxlin@pku.edu.cn

${ }^{\dagger}$ Equal contributors

${ }^{2}$ Department of Central Laboratory, School of Stomatology, Peking University, Beijing 100081, People's Republic of China

'Departments of Orthodontics and Central Laboratory, School of Stomatology, Peking University, \#22 Zhongguancun South Road, Haidian District, Beijing 100081, People's Republic of China

Full list of author information is available at the end of the article
}

the effect of orthodontic treatment [4]. The aim of this study was to determine the differences between periodontal and non-periodontal orthodontic patients.

Bacterial plaque is an etiologic factor in periodontitis [5]. The pathogenesis of this disease in adults results in a loss of connective tissue, bone support, and, ultimately, teeth [6]. The diagnosis of periodontitis and identification of at-risk patients are challenging. Periodontal condition is usually determined visually by clinical periodontal and X-ray examinations, which are subject to considerable measurement error due to clinical experience, and are often poorly tolerated by patients.

Saliva is a complex hypotonic hydrated solution [7] that contains 3397 different proteins with a variety of biological functions [8]. Whole saliva comprises the secretions of the major and minor saliva glands, nonsalivary gingival crevicular fluid, bronchial secretions, bacteria and bacterial products, deciduous epithelial 


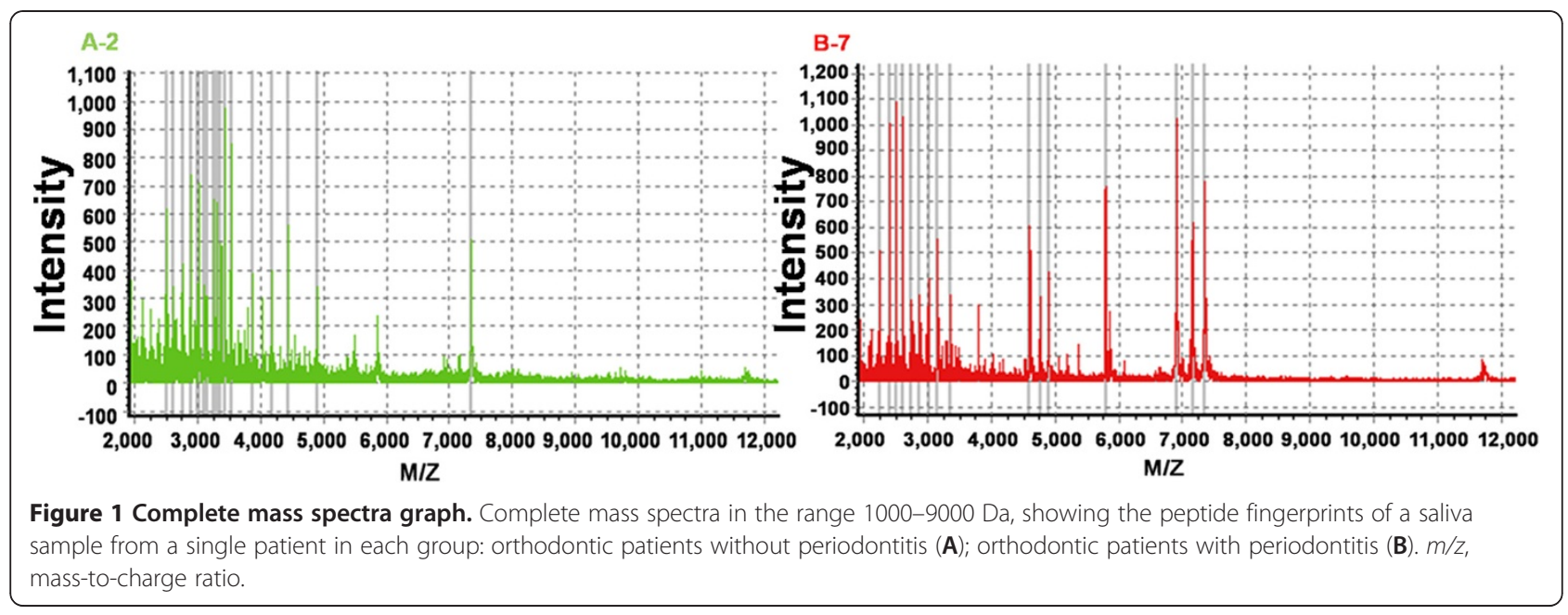

cells, and food debris. Moreover, saliva contains molecules which could be diffused or filtrated from blood [9]. For example, human C-reactive protein (CRP), which is used to assess the coronary events, could be present in saliva [10]. Periodontitis can involve the modified production or release of various factors, such as hormones and cytokines. We sought to discover a candidate biomarker that can facilitate the detection of periodontal changes before pathological symptoms occur.

Since saliva contains multiple potentially informative components and its collection is non-invasive, low-cost, and simple, research is increasingly focusing on the analysis of oral and systemic conditions using saliva as a detection strategy $[11,12]$. Moreover, there are databases (http://bioinformatics.ua.pt/OralCard/ and http://www. hspp.ucla.edu/) that compile proteomics data from oral cavity, contributing to the salivary analysis. And protein profiling methods such as two-dimensional polyacrylamide gel electrophoresis for separation and MS for identification have been used to investigate various conditions and disorders, including breast cancer [13], Sjögren's syndrome [14], rheumatoid arthritis [15], and oral pathologies such as oral cancer [16,17], dental caries [18], cleft palate [19], and periodontitis [20].

We used matrix-assisted laser desorption/ionization time-of-flight mass spectrometry (MALDI-TOF MS), a sensitive MS-based proteomic technique, to detect peptides over a large mass range. The mass spectra generated by this technique are easy to interpret [21]. Here, MALDI-TOF MS was used in combination with WCX to select peptides in the range of 1000-10000 Da prior to further identification. The effectiveness of this combination of techniques has been confirmed in many serum-based peptide profile identification studies [22,23]. MALDI-TOF MS generated accurate salivary protein profiles of patients with fixed orthodontic appliances with and without periodontitis.
In this study, the peptide mass profiles of saliva samples were investigated using magnetic bead-based peptidome analysis. In this way, we aimed to identify a panel of differentially-expressed specific candidate biomarkers.

\section{Results}

To investigate the differences between periodontalorthodontic and non-periodontal orthodontic patients, the entire mass spectra of the extracted peptide samples from 24 subjects (eight per group) were obtained by MALDI-TOF MS (Figure 1). Saliva peptidome fingerprint peaks were characterized in each patient by showing the maximum intensity within a particular $\mathrm{m} / \mathrm{z}$ range. The molecular weight of the majority of the peptides was 1000-7000 Da. The mass spectra peaks were then quantified and compared.

An average of 109 protein mass peaks was found. The peak intensities of nine $(1062.1,1454.2,2213.2,2621.9$, 3016.1, 3154.4, 3163.4, 5378.5, and 5435.2 Da) differed

Table 1 Significant $(p<0.05) m / z$ values discriminating samples from the two groups

\begin{tabular}{lcc}
\hline Mean $\boldsymbol{m} / \mathbf{z}$ value & $\boldsymbol{p}$-value & Tendency* \\
\hline 3163.4 & 0.008 & $\downarrow$ \\
1454.2 & 0.009 & $\uparrow$ \\
3154.4 & 0.012 & $\downarrow$ \\
2621.9 & 0.013 & $\downarrow$ \\
5378.5 & 0.023 & $\downarrow$ \\
1062.1 & 0.035 & $\uparrow$ \\
3016.1 & 0.042 & $\downarrow$ \\
5435.2 & 0.042 & $\downarrow$ \\
2213.2 & 0.045 & $\downarrow$
\end{tabular}

*Tendency, $m / z$ value intensity trend between the two groups: $\uparrow$, higher intensity in group $B$ than $A$; $\downarrow$, lower intensity in group $B$ than $A . m / z$, mass-tocharge ratio. $A$, the orthodontic patients without periodontitis; $B$, the orthodontic patients with periodontitis. 
significantly among the three different groups (Table 1 ). Orthodontic patients in the group without periodontal disease showed higher mass peaks for peptides of 2213.2, 2621.9, 3016.1, 3154.4, 3163.4, 5378.5, and $5435.2 \mathrm{Da}$, whereas the mass peaks for the peptides of 1062.1 and 1454.2 Da were higher in the periodontalorthodontic patients (Figures 2 and 3 ).

The most significant differences were exhibited by the 3163.4- and 1454.2-Da proteins $(p<0.01)$; the fit of the other combinations was not as good. Thus, we chose these two peptides to establish a fitted curve between the two groups (Figure 4). Samples from the two groups were well-separated, indicating that the fitting results were satisfactory.

Moreover, using LTQ-Orbitrap-MS detection, eight (1062.1, 1454.2, 2213.2, 2621.9, 3016.1, 3154.4, 3163.4, 5378.5 , and $5435.2 \mathrm{Da}$ ) of the nine differentiallyexpressed peptides were successfully identified (Table 2).
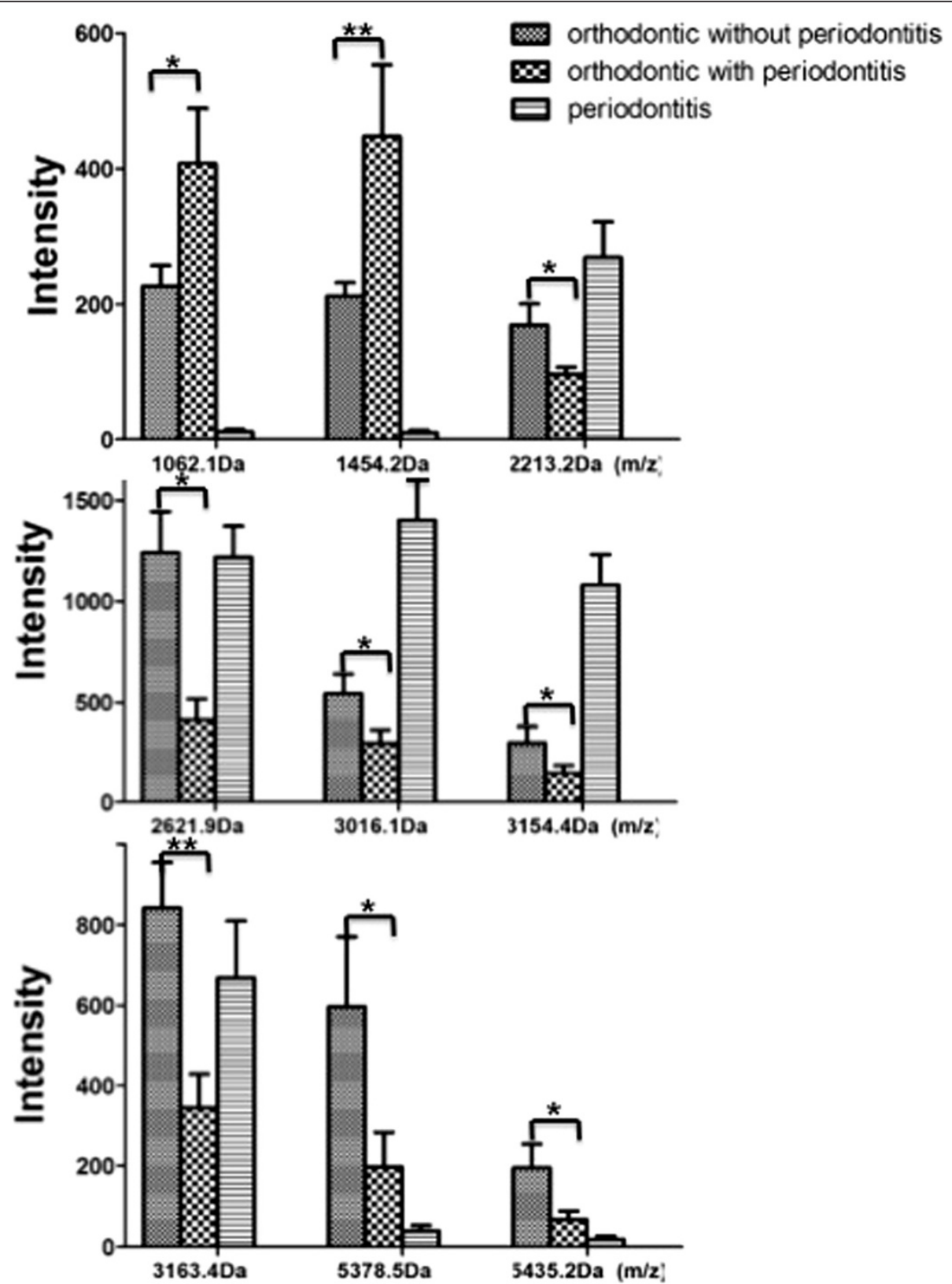

Figure 2 Column view of the mass spectra of the three groups. The peak intensities of the three different groups showed differential expression of nine salivary peptides. Noticeably, significant differences were investigated between the two orthodontic groups. $\left(^{*} p<0.05\right.$; $\left.{ }^{* *} p<0.01\right)$. 


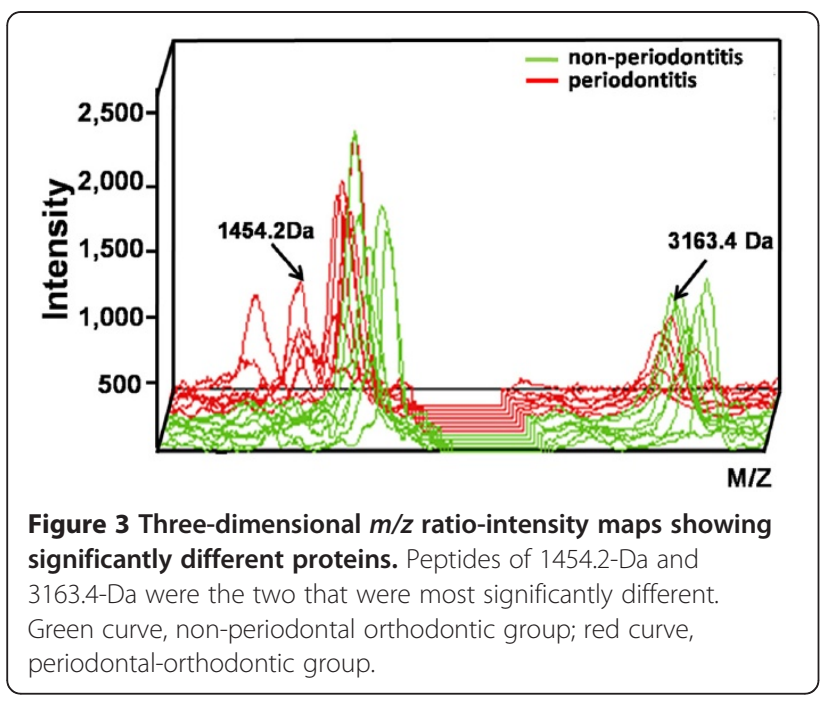

\section{Discussion}

Adult patients with periodontal disease exhibit tooth malocclusions such as flaring of the anterior segment. This unaesthetic appearance and/or dysfunction are the primary reasons for undergoing periodontal-orthodontic treatment using a multidisciplinary approach [24]. Combined orthodontic and periodontal treatment does not compromise the therapeutic effectiveness of the latter [25]. Indeed, this combination may be beneficial since it leads to increased bone resorption stability, a lack of incisor flaring, and improvements in bone defects [26]. However, some authors argue that orthodontic treatment is unsuitable in chronic periodontal patients because bacterial plaque is an etiologic factor in the development of periodontitis and the presence of orthodontic appliances facilitates plaque growth and maturation. Thus, the periodontal condition could worsen after orthodontic treatment [27]. Therefore, we examined periodontal-orthodontic patients with a view to elucidating the differences between them and nonperiodontal orthodontic patients.

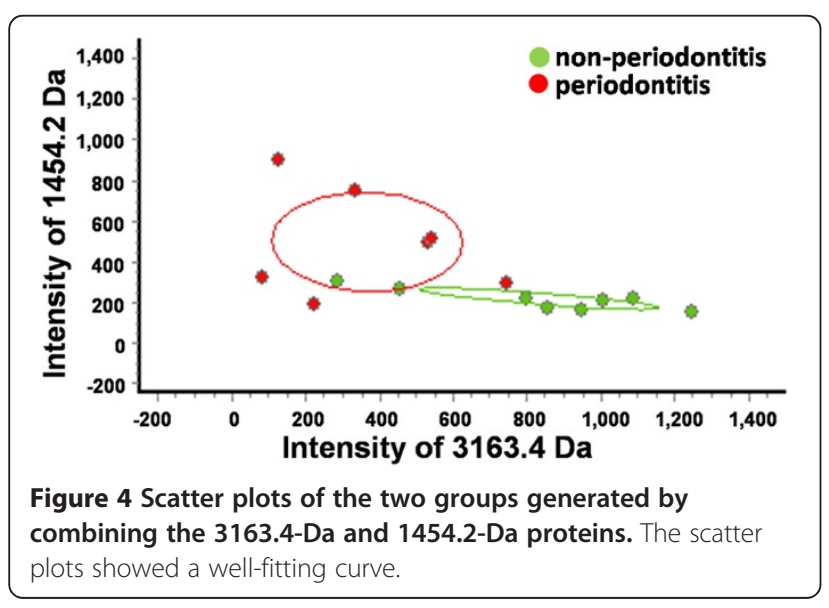

Such differences could be caused by inflammatory factors related to periodontitis or other protein factors [28], which may represent candidate biomarkers. A candidate biomarker is defined as an informative signal associated with a specific condition. The specificity and sensitivity of a biomarker describe its usefulness in diagnosing a specific condition or predicting its progress [29]. An effective biomarker should be measurable in an accessible body fluid, such as serum, urine, or saliva [30]. Saliva contains abundant proteins, peptides, and other small molecules [8]. Thus, the salivary peptide spectrum may be applied widely in the diagnosis and monitoring of oral diseases. Progress has been made in screening for not only oral diseases such as oral cancer [17], but also systematic conditions such as gastric cancer [31] and breast cancer [13]. Studies of periodontitis using proteomics $[32,33]$ have been performed, and the demand for periodontal-orthodontic combination treatment is increasing. However, little research on the salivary peptide spectrum in the periodontal-orthodontic area has been conducted.

In the present study, we used MALDI-TOF MS-based proteomic methods and WCX magnetic beads to examine all 24 saliva samples. Nine peaks that differed significantly were found (Figure 2), of which two (3163.4 and $1454.2 \mathrm{Da}$ ) exhibited the most significant differences $(p<0.01)$. Orthodontic treatment is a prolonged procedure. And in our previous study, alterations were found in salivary proteins due to different orthodontic treatment durations [34]. In this study, the peak intensities of the three different groups showed differential expression of salivary peptides, indicating the orthodontic treatment could contribute to the change of salivary peptidome. Noticeably, significant differences were investigated between the two orthodontic groups. This suggested that differential expression of salivary peptidome profile did exist between orthodontic patients with and without periodontitis. Thus, this method provides a new tool for analyzing the effect of periodontitis on orthodontic treatment.

The peptide sequence identifications made in this study have led to interesting speculations. The 3163.4Da peptide was identified as F2 prothrombin precursor. Thrombin is a 'trypsin-like' serine protease protein encoded by the F2 gene. Beyond its key role in the dynamic process of thrombus formation, thrombin has the potential to exert actions such as inflammation and leukocyte recruitment [35]. Moreover, isoform 1 of fibrinogen alpha chain precursor (FGA), which was the predicted identity of the 3154.4- and 2621.9-Da peptides, is encoded by FGA. It is cleaved by thrombin to form fibrin, indicating that it interacts with thrombin. Some studies [36] suggested epithelial thrombomodulin (TM), which binds to thrombin and converts it from a 
Table 2 Sequences of the differentially-expressed peptides

\begin{tabular}{|c|c|c|}
\hline Mean $\mathrm{m} / \mathrm{z}$ value & Peptide name & Peptide sequence \\
\hline 3163.4 & F2 Prothrombin precursor & LRPLFEKKSLEDKTERELLESYIDGR \\
\hline 1454.2 & SERPINA1(PRO2275) & PLFMGKWNPTQK \\
\hline 3154.4 & FGA Isoform 1 of fibrinogen alpha chain precursor & LIGQIVSSITASLR \\
\hline 2621.9 & FGA Isoform 1 of fibrinogen alpha chain precursor & SYKM*ADEAGSEADHEGTHSTKRGHAKSRP \\
\hline 5378.5 & $\begin{array}{l}\text { WWCE Isoform } 1 \text { of von Willebrand factor } C \text { and } \\
\text { EGF domain-containing protein precursor }\end{array}$ & APVNCSSCPGPPTASPSRPVLHLLQLLLRTNLM*KTQTLPTSPAGAHGPHSLA \\
\hline 1062.1 & $\begin{array}{c}\text { ITIH4 Isoform } 2 \text { of inter-alpha-trypsin inhibitor heavy } \\
\text { chain } \mathrm{H} 4 \text { precursor }\end{array}$ & SEMWAGKLQ \\
\hline 3016.1 & 280-kDa protein & LGVSPPPGAVLVLHSLPLEFPLAM*AFAEQ \\
\hline 5435.2 & Unknown peptide-identification failure & \\
\hline 2213.2 & ACTB Actin, cytoplasmic 1 & DLYANTVLSGGTTMYPGIADR \\
\hline
\end{tabular}

$\mathrm{m} / \mathrm{z}$, mass-to-charge ratio.

The English in this document has been checked by at least two professional editors, both native speakers of English. For a certificate, please see: http://www.textcheck.com/certificate/OlkIGD.

procoagulant protease to an anticoagulant enzyme, increased in gingival crevicular fluid of individuals with chronic periodontitis. Moreover, gingipains, the major periodontopathic bacterium Porphyromonas gingivalisderived cysteine proteases, lead to the degradation of endothelial TM. In addition, reduced expression of TM was found in gingival microvascular endothelia in patients with periodontitis [37], and this may be involved in the pathogenesis of periodontitis. Thus, these differential expression patterns of altered proteins may have originated from periodontitis-associated inflammation or differences in bone metabolism between orthodontic patients with and without periodontitis. Ultimately, our aim is to determine the protein or gene from which a peptide is derived; however, this is complex. When the distribution of the peptides with low molecular weight were intended to match to the mass spectrometry spectra relative peak area, it should be noted that these peptides had complicated origins. Peptides in saliva could be secreted peptides and proteolytic fragments of related proteins [38]. Moreover, these components are subject to secondary modifications from distinctive protein families. Thus, a peptide sequence usually does not exclusively define a single protein.

As these specific peptides were investigated using this relatively new combined method with no validation using other techniques, thus, a larger sample size and repeated trial or trial using other techniques are needed to confirm the significant differences in peptide mass peaks found in this study and its reproducibility. The establishment of a relatively complete protein-peptide spectrum database will facilitate the determination of both the source of the salivary protein profile variation and the mechanism thereof. Moreover, the analysis of saliva is inherently challenging because of the wide protein concentration range therein [29] and the presence of multiple post-translational modifications [39]. Further research into the composition of saliva will likely provide novel tools for investigations of physiological and pathophysiological states.

\section{Conclusions}

In conclusion, our data suggest that the salivary protein profiles of periodontal, non-periodontal orthodontic patients and periodontitis patients exhibit differential mass spectra peak intensities (i.e., the peptide profile is altered in cases of periodontitis). This represents a new method of analyzing the effect of periodontitis on orthodontics and orthodontic contribution to the alterations of salivary peptidome profile, ultimately leading to increased treatment efficacy. However, expansion of the orthodontic patient dataset and the identification of additional candidate biomarkers are necessary to establish an effective diagnosis and monitoring model for periodontal-orthodontic treatment.

\section{Methods}

\section{Ethics statement}

This study was approved by the Peking University Biomedical Ethics Committee. Adult subjects and parents of pediatric subjects signed an informed consent form before participating in the study.

\section{Subjects}

Patients seeking treatment at the Stomatology School of Peking University were recruited in November, 2011. All 24 study subjects were generally ensured systemically healthy via testing of serum basic biochemical items and inquiring medical history, and those who presented with caries, diseases of the oral mucosa, or oral cancers were excluded. Recorded clinical periodontal parameters of the subjects included bleeding on probing (BOP),probing 
pocket depth (PPD) and clinical attachment level (CAL). Chronic periodontitis was diagnosed in patients who had six sites BOP on different teeth with $\mathrm{PPD} \geq 6 \mathrm{~mm}$ and $\mathrm{CAL}>5 \mathrm{~mm}$. Control orthodontic patients had $<10 \%$ of sites BOP and $\mathrm{PPD}<3 \mathrm{~mm}$. And all the periodontitis patients manifested to be moderate degree of chronic periodontitis. Then the patients were divided into three groups according; that is, periodontalorthodontic, non-periodontal orthodontic groups and patients with periodontitis but no orthodontic treatment. The characteristics of the subjects are presented in Table 3.

The treatment procedure for all subjects involved the use of a fixed appliance, followed by alignment, leveling, and refinement procedures. All patients were asked to maintain good oral hygiene during treatment and were counseled regarding oral hygiene and tooth brushing both before treatment and at each visit.

\section{Saliva collection and processing}

All individuals were asked to rest for $15 \mathrm{~min}$ before saliva collection at $8: 30 \mathrm{am}$, and not to eat or drink after dinner the previous evening or to brush their teeth on the collection day morning. The subjects sat upright in a quiet room and were required to put the tip of their tongue against the sublingual caruncle without straining. Thus, the saliva, which was received in a paper cup for the first $5 \mathrm{~min}$, could run from the mouth, and we collected $6 \mathrm{~mL}$ of the spontaneous saliva flow in a $50-\mathrm{mL}$ centrifuge tube. During the collection procedure, patients were asked not to speak. Immediately after collection, the unstimulated whole saliva samples were kept on ice and then centrifuged at $9000 \times g$ for $7 \mathrm{~min}$ at $4^{\circ} \mathrm{C}$ to remove insoluble materials, cells, and debris. Ethylene diamine tetraacetic acid $(1 \mathrm{mM}$; Sigma, St. Louis, MO, USA) and $1 \mathrm{mM}$ phenylmethyl sulfonylfluoride (Sigma) were added to the sample supernatants to inhibit protease activity. Protein concentrations were determined using the Lowry assay and ELx808 Protein Assay (BioTek, Hercules, CA, USA). The supernatants were kept at $-80^{\circ} \mathrm{C}$ for further analysis.

\section{Reagents and instruments}

The WCX magnetic bead kit (SPE-C; Bioyong Tech, Beijing, China), alpha-cyano-4-hydroxycinnamic acid (HCCA), MALDI-TOF MS (Bruker Bio-sciences, Bremen,

Table 3 Demographic information for the two groups

\begin{tabular}{llll}
\hline Group & Sample size & \multicolumn{2}{c}{ Age } \\
\cline { 2 - 4 } & & Mean & SD \\
\hline orthodontic patients without periodontitis & 8 & 35.4 & 1.3165 \\
orthodontic patients with periodontitis & 8 & 33 & 2.2509 \\
patients with periodontitis & 8 & 34.55 & 2.2181 \\
\hline
\end{tabular}

Germany), 100\% ethanol (chromatographic grade), and $100 \%$ acetone (chromatographic grade) were freshly prepared.

\section{WCX fractionation and MALDI-TOF MS}

The low-molecular-weight (LMW) salivary peptidome contains enormous and important biological information and they should be a rich source of specific candidate biomarkers [13]. Unlike 2D-PAGE analysis, which only allows the separation of proteins with molecular weight in a range of $20-300 \mathrm{kDa}$, the magnetic bead-based profiling MS technologies could cover a range of $1-250 \mathrm{kDa}$ [29]. Moreover, the high-throughput nature of MALDI-TOF MS makes fast screening for novel candidate biomarkers possible, and this method is cost effective and can be easily adopted [31]. Besides, this combined method only required $20 \mathrm{ul}$ processed sample for generating enough proteins peaks profiling by MALDI-TOF-MS.

The WCX magnetic bead kit suspension was mixed by shaking. After eluting and beating, the magnetic beads were separated from the protein and the eluted peptide samples were transferred to a clean $0.5-\mathrm{mL}$ sample tube for further analysis by MS.

Five microliters of HCCA substrate solution $(0.4 \mathrm{~g} / \mathrm{L}$, dissolved in acetone and ethanol) and 0.8-1.2 $\mu \mathrm{L}$ of eluate were mixed. Next, 0.8-1.2 $\mu \mathrm{L}$ of this mixture was applied to a metal target plate and dried at room temperature. Finally, the prepared samples were analyzed by MALDI-TOF MS. Peptides with a molecular weight in the range of 1000-10000 Da were collected, and 400 shots of laser energy were used. Peptide mass fingerprints were obtained by accumulating 50 single MS signal scans.

\section{Identification of differentially expressed candidate biomarkers}

The sequences of peptides expressed differentially between the two groups were determined by nano-liquid chromatography-electrospray ionization-tandem mass spectrometry (nano-LC/ESI-MS/MS) using a setup consisting of an Aquity UPLC system (Waters) and an LTQ Obitrap XL mass spectrometer (Thermo Fisher) equipped with a nano-ESI source. The obtained chromatograms were analyzed with BioworksBrowser 3.3.1 SP1, and the resulting mass lists were used in a database search with Sequest ${ }^{\mathrm{TM}}$ [IPI Human (3.45)]. The parameters used to generate the peak list were: parent ion and fragment mass relative accuracy $(50 \mu \mathrm{g} / \mathrm{g}$ and $1 \mathrm{Da}$, respectively).

\section{Statistical analysis}

A two-tailed $t$-test and Student's $t$-test were used to compare peptide peak intensities between the two groups. 
Data were analyzed using the BioExplorer statistical package (BioyongTech). A $p$-value $<0.05$ was considered significant.

\section{Competing interests}

The authors declare that they have no competing interests.

\section{Authors' contributions}

LX and CF conceived of the idea for the peptidomic study and participated in its design. ZJN and ZSN carried out a major portion of the data analysis and drafted the manuscript. LRX, ZH and WXD carried out the sample collection and extraction. $\mathrm{ZYH}$ and DN carried out the data statistical analysis and a portion of the data analysis. CT provided the orthodontic patients. All authors read and approved the final manuscript.

\section{Acknowledgements}

The authors gratefully acknowledge the assistance of Dr. Yanqiu Zhou of Peking University School of Stomatology for her research suggestions, Dr. Yan Li of BioyongTech for her technical support, and Jianguo Ji (Peking University) for his expertise in mass spectrometry. This study was supported by funding from Peking University School of Stomatology (PKUSS20110301).

\section{Author details}

Departments of Orthodontics and Central Laboratory, School of Stomatology, Peking University, \#22 Zhongguancun South Road, Haidian District, Beijing 100081, People's Republic of China. ${ }^{2}$ Department of Central Laboratory, School of Stomatology, Peking University, Beijing 100081, People's Republic of China. ${ }^{3}$ Department of Stomatology, Beijing An Zhen Hospital, Capital University of Medical Sciences, Beijing 100081, People's Republic of China.

Received: 5 July 2012 Accepted: 23 October 2012

Published: 6 November 2012

\section{References}

1. Kim Yl, Kim MJ, Choi Jl, Park SB: A multidisciplinary approach for the management of pathologic tooth migration in a patient with moderately advanced periodontal disease. Int I Periodontics Restorative Dent 2012, 32:225-230.

2. Zetu I, Ogodescu E, Zetu L, Stratul SI, Rusu D, Talpos S, Ogodescu A: [An interdisciplinary approach to complex orthodontic-periodontal clinical situations in adult patients]. Rev Med Chir Soc Med Nat lasi 2011, 115:1262-1266.

3. Tew JG, El Shikh ME, El Sayed RM, Schenkein HA: Dendritic cells, antibodies reactive with oxLDL, and inflammation. J Dent Res 2012, 91:8-16.

4. Dersot JM: Gingival recession and adult orthodontics: a clinical evidencebased treatment proposal. Int Orthod 2012, 10:29-42.

5. Beader N, Ivic-Kardum M: The role of cytomegalovirus infection in the pathogenesis of periodontal diseases. Acta Clin Croat 2011, 50:61-66.

6. Tezal M, Uribe S: A lack of consensus in the measurement methods for and definition of periodontitis. J Am Dent Assoc 2011, 142:666-667.

7. Bader HI: Salivary diagnostics in medicine and dentistry: a review. Dent Today 2011, 30:46-48. 50-41; quiz 52-43.

8. Rosa N, Correia MJ, Arrais JP, Lopes P, Melo J, Oliveira JL, Barros M: From the salivary proteome to the OralOme: comprehensive molecular oral biology. Arch Oral Biol 2012, 57:853-864.

9. Punyadeera C, Dimeski G, Kostner K, Beyerlein P, Cooper-White J: One-step homogeneous C-reactive protein assay for saliva. J Immunol Methods 2011, 371:19-25

10. Punyadeera C: Human saliva as a tool to investigate intimate partner violence. Brain Behav Immun 2012, 26:541-542.

11. Li Y, Denny P, Ho CM, Montemagno C, Shi W, Qi F, Wu B, Wolinsky L, Wong DT: The Oral Fluid MEMS/NEMS Chip (OFMNC): diagnostic and translational applications. Adv Dent Res 2005, 18:3-5.

12. Schulz BL, Cooper-White J, Punyadeera C: Saliva proteome research: current status and future outlook. Crit Rev Biotechnol 2012 [Epub ahead of print].

13. Zhang L, Xiao H, Karlan S, Zhou H, Gross J, Elashoff D, Akin D, Yan X, Chia D, Karlan B, Wong DT: Discovery and preclinical validation of salivary transcriptomic and proteomic biomarkers for the non-invasive detection of breast cancer. PLoS One 2010, 5:e15573.
14. Ferraccioli G, De Santis M, Peluso G, Inzitari R, Fanali C, Bosello SL, lavarone F, Castagnola M: Proteomic approaches to Sjogren's syndrome: a clue to interpret the pathophysiology and organ involvement of the disease. Autoimmun Rev 2010, 9:622-626.

15. Giusti L, Baldini C, Ciregia F, Giannaccini G, Giacomelli C, De Feo F, Delle Sedie A, Riente L, Lucacchini A, Bazzichi L, Bombardieri S: Is GRP78/BiP a potential salivary biomarker in patients with rheumatoid arthritis? Proteomics Clin Appl 2010, 4:315-324.

16. Wu JY, Yi C, Chung HR, Wang DJ, Chang WC, Lee SY, Lin CT, Yang YC, Yang WC: Potential biomarkers in saliva for oral squamous cell carcinoma. Oral Oncol 2010, 46:226-231.

17. Hu S, Arellano M, Boontheung P, Wang J, Zhou H, Jiang J, Elashoff D, Wei R, Loo JA, Wong DT: Salivary proteomics for oral cancer biomarker discovery. Clin Cancer Res 2008, 14:6246-6252.

18. Vitorino R, de Morais Guedes S, Ferreira R, Lobo MJ, Duarte J, Ferrer-Correia AJ, Tomer KB, Domingues PM, Amado FM: Two-dimensional electrophoresis study of in vitro pellicle formation and dental caries susceptibility. Eur J Oral Sci 2006, 114:147-153.

19. Szabo GT, Tihanyi R, Csulak F, Jambor E, Bona A, Szabo G, Mark L: Comparative Salivary Proteomics of Cleft Palate Patients. Cleft Palate Craniofac J 2011, 48:708-716.

20. Goncalves Lda R, Soares MR, Nogueira FC, Garcia C, Camisasca DR, Domont G, Feitosa AC, Pereira Dde A, Zingali RB, Alves G: Comparative proteomic analysis of whole saliva from chronic periodontitis patients. J Proteomics 2010, 73:1334-1341.

21. Al-Tarawneh SK, Bencharit S: Applications of Surface-Enhanced Laser Desorption/lonization Time-Of-Flight (SELDI-TOF) Mass Spectrometry in Defining Salivary Proteomic Profiles. Open Dent J 2009, 3:74-79.

22. Schaub NP, Jones KJ, Nyalwidhe JO, Cazares LH, Karbassi ID, Semmes OJ, Feliberti EC, Perry RR, Drake RR: Serum proteomic biomarker discovery reflective of stage and obesity in breast cancer patients. J Am Coll Surg 2009, 208:970-978. discussion 978-980.

23. Schwamborn K, Krieg RC, Grosse J, Reulen N, Weiskirchen R, Knuechel R, Jakse G, Henkel C: Serum proteomic profiling in patients with bladder cancer. Eur Urol 2009, 56:989-996.

24. Derton N, Derton R, Perini A, Gracco A, Fornaciari PA: Orthodontic treatment in periodontal patients: a case report with 7 years follow-up. Int Orthod 2011, 9:92-109.

25. Lee JW, Lee SJ, Lee CK, Kim BO: Orthodontic treatment for maxillary anterior pathologic tooth migration by periodontitis using clear aligner. J Periodontal Implant Sci 2011, 41:44-50.

26. Boyer S, Fontanel F, Danan M, Olivier M, Bouter D, Brion M: Severe periodontitis and orthodontics: evaluation of long-term results. Int Orthod 2011, 9:259-273.

27. van Gastel J, Teughels W, Quirynen M, Struyf S, Van Damme J, Coucke W, Carels C: Longitudinal changes in gingival crevicular fluid after placement of fixed orthodontic appliances. Am J Orthod Dentofacial Orthop 2011, 139:735-744.

28. Hans $\mathrm{M}$, Hans VM: Toll-like receptors and their dual role in periodontitis: a review. J Oral Sci 2011, 53:263-271.

29. Al-Tarawneh SK, Border MB, Dibble CF, Bencharit S: Defining salivary biomarkers using mass spectrometry-based proteomics: a systematic review. OMICS 2011, 15:353-361.

30. Yang J, Song YC, Dang CX, Song TS, Liu ZG, Guo YM, Li ZF, Huang C: Serum peptidome profiling in patients with gastric cancer. Clin Exp Med 2011, 12 (2):79-87.

31. Wu ZZ, Wang JG, Zhang XL: Diagnostic model of saliva protein finger print analysis of patients with gastric cancer. World I Gastroenterol 2009, $15: 865-870$

32. Mizuno N, Niitani M, Shiba H, Iwata T, Hayashi I, Kawaguchi H, Kurihara H: Proteome analysis of proteins related to aggressive periodontitis combined with neutrophil chemotaxis dysfunction. J Clin Periodontol 2011, 38:310-317.

33. Rangé H, Léger T, Huchon C, Ciangura C, Diallo D, Poitou C, Meilhac O, Bouchard P, Chaussain C: Salivary proteome modifications associated with periodontitis in obese patients. J Clin Periodontol 2012, 39:799-806

34. Zhang J, Zhou S, Zheng H, Zhou Y, Chen F, Lin J: Magnetic bead-based salivary peptidome profiling analysis during orthodontic treatment durations. Biochem Biophys Res Commun, 421:844-849.

35. O'Brien M: The reciprocal relationship between inflammation and coagulation. Top Companion Anim Med 2012, 27:46-52. 
36. Matsuyama T, Tokuda M, Izumi Y: Significance of thrombomodulin release from gingival epithelial cells in periodontitis patients. $J$ Periodontal Res 2008, 43:379-385.

37. Inomata M, Ishihara Y, Matsuyama T, Imamura T, Maruyama I, Noguchi T, Matsushita K: Degradation of vascular endothelial thrombomodulin by arginine- and lysine-specific cysteine proteases from Porphyromonas gingivalis. J Periodontol 2009, 80:1511-1517.

38. Amado F, Lobo MJ, Domingues P, Duarte JA, Vitorino R: Salivary peptidomics. Expert Rev Proteomics 2010, 7:709-721.

39. Pfaffe T, Cooper-White J, Beyerlein P, Kostner K, Punyadeera C: Diagnostic Potential of Saliva: Current State and Future Applications. Clin Chem 2011, 57:675-687.

doi:10.1186/1477-5956-10-63

Cite this article as: Zhang et al:: Magnetic bead-based salivary

peptidome profiling for periodontal-orthodontic treatment. Proteome Science 2012 10:63.

\section{Submit your next manuscript to BioMed Central and take full advantage of:}

- Convenient online submission

- Thorough peer review

- No space constraints or color figure charges

- Immediate publication on acceptance

- Inclusion in PubMed, CAS, Scopus and Google Scholar

- Research which is freely available for redistribution 